\title{
How do we quantify biodiversity? \\ All the evidence in one place
}

Olga Lyashevska

olyashevska01@qub.ac.uk

Abstract
Biodiversity is a multi-dimensional concept that is represented by a large variety of
measures. This complexity and lack of consistency limits the development of a co-
herent scientific understanding of biodiversity and how properties, such as ecosystem
services, may depend on it. Here, I demonstrate that the formal discipline of creating
a relational database (RDB) for information about biodiversity and its measures, is a
useful tool in organising such knowledge into coherent sense. Following steps of the
logical database design and data normalization to build a RDB, results in a formal def-
inition of biodiversity within a well defined concept structure; mapping rules between
the concepts of biodiversity and entities of RDB and a consistent information structure
- all in one place. I show how this is then used to support evidence-based objective
statements about biodiversity.

\section{Concept definition}

A database of measures of biodiversity will be constructed. It will be used to answer the following question:

'To what extent does existing knowledge describe the information content (=fundamental biodiversity) of biological systems?'

Fundamental biodiversity is the set of differences in measures $M$ among a set of biological systems $(i, j)$ whose component measures are strictly orthogonal.

Measure $M_{D L} \equiv(D \mid L)$ is a scalar combination of one descriptor $\mathbf{D}$ at one level $\mathrm{L}$ specifying a component of biodiversity. The matrix measure $\mathbf{M}$ is the set of all possible combinations $M_{D L}(\forall D, L)$.

N.B. $M_{D L}$ can be null, so that $\mathbf{M}$ may be a sparse matrix.

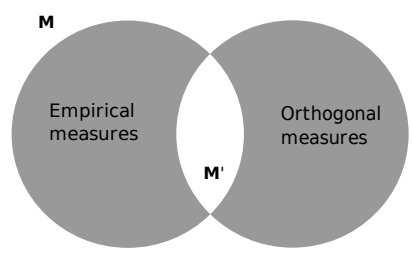

Empirical measures are measures collected from the literature

Orthogonal measures are hypothetically possible measures that fully describe biodiversity;

Intersect $M^{\prime}$ is a description of biodiversity covered by literature, other sectors are unnecessary or missing;

\section{Database specification}

A relational database of measures of biodiversity biodivDB is running on a remote server. It can be accessed via both command line and phpMyAdmin. It contains 13 tables; the storage engine is InnoDB. To facilitate analysis of database it can be connected with R using RMySQL package.

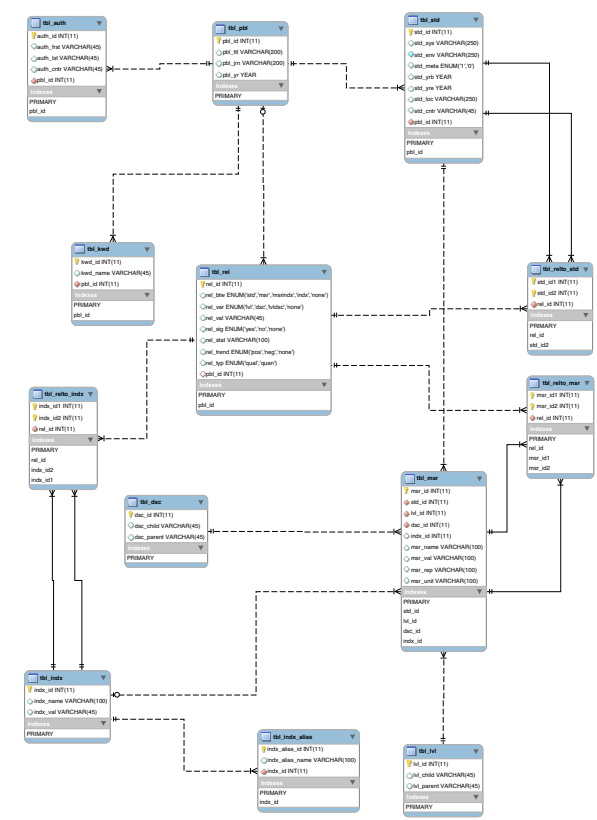

The database will help to reveal patterns among biological systems and imply the minimum set of orthogonal measures.

\begin{tabular}{ll|ll}
\multicolumn{4}{c}{ Data dictionary } \\
\hline Table name & Description & Table name & Description \\
\hline tbl_auth & author & tbl_pbl & publication \\
tbl_std & study & tbl_kwd & keyword \\
tbl_rel & relationship & tbl_tbl_relto_std & related to study \\
tbl_tbl_relto_indx & related to index & tbl_relto_msr & related to measure \\
tbl_dsc & descriptor & tbl_msr & measure \\
tbl_indx & index & tbl_indx_alias & index alias \\
tbl_lvl & level & & \\
\hline
\end{tabular}

\section{Cartesian Product $M_{D L} \equiv(D \mid L)$}

Cartesian product is a way to extract matrix elements of $\mathbf{M}$, so a view of cartesian join will be created. Every row of table tbl_lvl is joined with every row of tbl_dsc. This join produces a matrix of elements $(D \mid L)$, that is a matrix of all hypothetically possible measures made from descriptors $D$ and a given level $L$.

CREATE VIEW tbl_cart1 AS SELECT lvl_child, dsc_child

FROM tbl_lvl, tbl_dsc

It generates 598 rows where each row is an element of the $M_{D L}$ matrix. To compare generated measures with measures actually present in tbl_msr I run the following query:

CREATE VIEW tbl_cart2

AS SELECT distinct dsc_child, lvl_child, msr_name

FROM tbl_cart1 LEFT JOIN tbl_msr

ON cart1.dsc_id=tbl_msr.dsc_id AND cart1.lvl_id=tbl_msr.lvl_id

WHERE msr_name IS NOT NULL;

It generates 45 rows, which are in fact distinct measures of biodiversity presented in the database. Analogically I can find a set of values where msr_name IS NULL. The difference between the two sets will indicate measures that are $\mathrm{M}_{D L}=\emptyset .518$ rows or $92 \%$ of all hypothetically possible measures are missing.

\section{Preliminary Results}

- database contains 30 publications, 53 studies (31 distinct) and 189 (43 distinct) measures of biodiversity;

- word 'diversity' appears 134 times in title or keywords in the period from 1995 to 2009.

- number of measures increases at a rate greater than the number of studies with an increase in a number of publications;
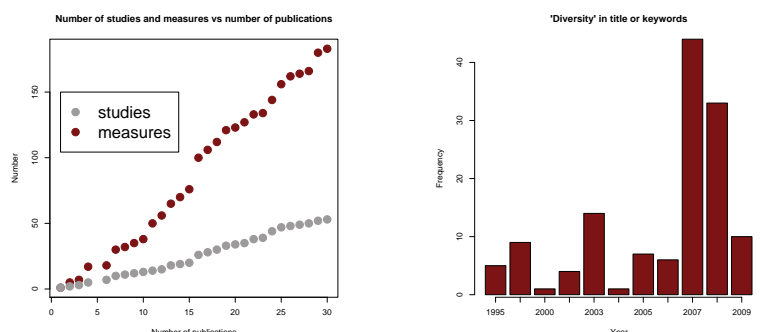

- 103 measures provide a value of measure, corresponding to 15 levels and 17 descriptors;

- 95 measures out of 189 are used to create an index (49 distinct pairs);

- species richness and species abundance are the most frequently used measures (46 and 38 respectively);

- systems that are mostly measured are grassland (34), insects (33), and macrobenthos (22);

- most of the relationships established are on measures level (48);

\section{Further Work}

At the point when an additional publication will not produce any new empirical measures, it is said that the saturation point has been reached. When saturation point is identified I will quantify the Venn diagram. If the rate of growth of publication/measure ratio of biodiversity is known, the number of measures needed to fully describe the information content of fundamental biodiversity can be predicted with greater confidence.

\section{Acknowledgements}

I am grateful to Dr Keith Farnsworth for his supervision and EPA in Ireland for funding 